\title{
Design of experiments: a statistical tool for PIV uncertainty quantification
}

\section{S. Adatrao ${ }^{1 *}$, A. Sciacchitano ${ }^{1}$, S. van der Velden ${ }^{1}$, M. J. van der Meulen', M. Cruellas Bordes ${ }^{3}$}

\author{
${ }^{1}$ Department of Aerospace Engineering, Delft University of Technology, Delft, The Netherlands \\ ${ }^{2}$ Royal Netherlands Aerospace Center (NLR), Marknesse, The Netherlands \\ ${ }^{3}$ German-Dutch Wind Tunnels (DNW), Marknesse, The Netherlands \\ *s.adatrao@tudelft.nl
}

\begin{abstract}
A statistical tool called Design of Experiments (DOE) is introduced for uncertainty quantification in particle image velocimetry (PIV). DOE allows to quantify the total uncertainty as well as the systematic uncertainties arising from various experimental factors. The approach is based on measuring a quantity (e.g. time-averaged velocity from PIV) several times by varying the levels of the experimental factors which are known to affect the value of the measured quantity. In this way, using Analysis of Variances (ANOVA), the total variance in the measured quantity can be computed and hence the total uncertainty. Moreover, the analysis provides the individual variances for each of the experimental factors leading to the estimation of the systematic uncertainties from each factor and their contribution to the total uncertainty. The methodology is assessed for an experimental test case of the flow at the outlet of a ducted Boundary Layer Ingesting (BLI) propulsor to quantify the total uncertainty in time-averaged velocity from stereoscopic PIV measurements as well as the constituent systematic uncertainties due to the experimental factors, namely, camera aperture, inter-frame time separation, interrogation window size and stereoscopic camera angle.
\end{abstract}

\section{Introduction}

Despite the quantification of PIV uncertainty being the key to discern measurement errors from the true flow physics, PIV uncertainty quantification (UQ) is often hindered by the complexity of the measurement chain, which introduces errors from various sources such as particles, illumination, imaging and processing. Several approaches have been proposed for PIV uncertainty quantification. However, they mostly focused on quantifying the uncertainty from random errors and were limited in the quantification of the systematic uncertainty (Sciacchitano 2019). Moreover, the main results of the $4^{\text {th }}$ international PIV challenge (Kähler et al. 2016) showed that, even for the same set of image recordings, large differences in the PIV results occurred among the participants due to the selection of the different processing parameters. The systematic error sources in PIV are not only significant in the processing stage, but also during the data acquisition (Sciacchitano 2019, among others). Thus, it is necessary to quantify the constituent uncertainties arising from various systematic error sources while calculating the total uncertainty in PIV measurements.

Design of Experiments (DOE) is a statistical tool used in many fields of science and engineering to evaluate the systematic effect of input factors on the measurement output. It was first employed for wind tunnel measurements by DeLoach (2000) and was shown to be effective for quantifying the systematic errors in experiment (DeLoach et al. 2012). Smith and Oberkampf (2014) stated DOE to be an alternative tool to 
overcome the limitations of the traditional UQ methods. Beresh (2009) and DeBonis et al. (2012) made use of DOE to quantify the uncertainty of their PIV measurements in a transonic and supersonic flow, respectively. However, their UQ analysis was conducted only at the intersection points of two measurement planes. In this work, a PIV-UQ approach based on DOE and Analysis of Variances (ANOVA) is introduced and demonstrated for stereoscopic PIV measurements at the outlet of a ducted Boundary Layer Ingesting (BLI) propulsor.

\section{Methodology}

DOE refers to the process of planning the experiment in order to collect appropriate data that can be analysed by statistical methods resulting in valid and objective conclusions (Montgomery 2013). In any experiment, some of the experimental parameters directly affect the output value and are called design factors; in PIV measurements, those are for instance the inter-frame time separation, interrogation window size, camera aperture, stereoscopic camera angle, etc. Additionally, some of the parameters, which affect the output directly or indirectly (in combination with the design factors) but are uncontrollable (or only partly controllable) during the measurements, are called nuisance factors; for PIV, those include variations of the fluid properties during a measurement, seeding density and its distribution, etc. Different measurements of a constant (ideally) quantity with varying levels of the design and/or nuisance factors show variations in the measured quantity. A proper data acquisition model and statistical analysis can be used to quantify the variance in the output quantity due to the variations in the levels of input factors (and their combinations). The present work employs the statistical tools DOE and ANOVA to quantify the total uncertainty in time-averaged PIV measurements and the contribution of the design and nuisance factors to the total uncertainty. Following Montgomery (2013), a Randomized Complete Block Design (RCBD) is considered for data acquisition, as blocking is necessary for tackling the effect of the nuisance factors. In such experimental design, measurements are carried out in two or more blocks (of the nuisance factor), where the levels of the blocking factor are fixed in each block and the levels of the design factors are varied randomly in each block. Let us take an example of experiment with two design factors $A$ and $B$ with $a$ and $b$ number of levels, respectively, and one blocking factor with $n$ number of levels. Following Montgomery (2013), a linear statistical model for this design is:

$$
y_{i j k}=\mu+A_{i}+B_{j}+(A B)_{i j}+\text { Block }_{k}+\varepsilon_{i j k}
$$

where, $y_{i j k}$ is the observed response at the $i^{\text {th }}$ level of factor $A$ and $j^{\text {th }}$ level of factor $B$ in $k^{\text {th }}$ block, $\mu$ is the overall mean effect, $A_{i}$ is the effect of the $i^{\text {th }}$ level of factor $A, B_{j}$ is the effect of the $j^{\text {th }}$ level of factor $B,(A B)_{i j}$ is the effect of the interaction between $A_{i}$ and $B_{j}, B l o c k_{k}$ is the effect of the $k^{\text {th }}$ level of the blocking factor, and $\varepsilon_{i j k}$ is an error component consisting of random error and the effect of unknown factors (i.e. their first order and higher order main and interaction effects) in the measurements. For this model, we are interested in checking whether the effects $A_{i}, B_{j},(A B)_{i j}$ and $B l o c k_{k}$ are zero (null hypothesis) or non-zero (alternative hypothesis). This can be achieved by the factorial ANOVA (Montgomery 2013) as shown in table 1, where $y_{i .}$. denotes the total of all observations under the $i^{\text {th }}$ level of factor $A, y_{. j}$. denotes the total of all observations under the $j^{\text {th }}$ level of factor $B, y_{i j}$. denotes the total of all observations under the $i^{\text {th }}$ level of factor $A$ and $j^{\text {th }}$ level of factor $B, y_{. . k}$ denotes the total of all observations under the $k^{\text {th }}$ level of blocking factor, and $y_{\ldots . . .}$ denotes the grand total of all the observations. From the ANOVA table 1, the significance of the factor effects is determined by performing the $F$-test with desired confidence level, where the mean squares $(M S)$ of the effects are compared with that of the error mean square $\left(M S_{\varepsilon}\right)$. Therefore, it is possible to segregate the contribution of every factor in the total variance of the measurement. The total uncertainty $\left(U_{\text {Total }}\right)$ and constituent systematic uncertainties $\left(U_{X}\right)$ in the response variable are calculated as: 


$$
\begin{aligned}
U_{\text {Total }} & =\sqrt{\frac{S S_{\text {Total }}}{a b n-1}}=\sqrt{U_{A}^{2}+U_{B}^{2}+U_{A B}^{2}+U_{B l o c k}^{2}+U_{\varepsilon}^{2}} \\
U_{X} & =\sqrt{\frac{S S_{X}}{a b n-1}} \text { and } X=A, B, A B, \text { Block, } \varepsilon
\end{aligned}
$$

where, $S S$ is the sum of squares and represents the variability in the response variable as shown in the table 1.

Table 1: Analysis of Variances (ANOVA) table for Two-Factor Randomized Complete Block Design (RCBD)

\begin{tabular}{|c|c|c|c|c|}
\hline Source & Sum of Squares & $\begin{array}{c}\text { Degrees of } \\
\text { Freedom }\end{array}$ & Mean Squares & $\boldsymbol{F}_{\mathbf{0}}$ \\
\hline $\mathrm{A}$ & $S_{A}=\frac{1}{b n} \sum_{i} y_{i . .}^{2}-\frac{y_{\ldots}^{2}}{a b n}$ & $a-1$ & $M S_{A}=\frac{S S_{A}}{a-1}$ & $F_{0}=\frac{M S_{A}}{M S_{\varepsilon}}$ \\
\hline $\mathrm{B}$ & $S S_{B}=\frac{1}{a n} \sum_{j} y_{. j .}^{2}-\frac{y_{\ldots}^{2}}{a b n}$ & $b-1$ & $M S_{B}=\frac{S S_{B}}{b-1}$ & $F_{0}=\frac{M S_{B}}{M S_{\varepsilon}}$ \\
\hline $\mathrm{AB}$ & $S S_{A B}=\frac{1}{n} \sum_{i} \sum_{j} y_{i j .}^{2}-\frac{y_{\ldots}^{2}}{a b n}-S S_{A}-S S_{B}$ & $(a-1)(b-1)$ & $M S_{A B}=\frac{S S_{A B}}{(a-1)(b-1)}$ & $F_{0}=\frac{M S_{A B}}{M S_{\varepsilon}}$ \\
\hline Block & $S S_{B l o c k}=\frac{1}{a b} \sum_{k} y_{. . k}^{2}-\frac{y_{\ldots}^{2}}{a b n}$ & $n-1$ & $M S_{B l o c k}=\frac{S S_{B l o c k}}{n-1}$ & $F_{0}=\frac{M S_{B l o c k}}{M S_{\varepsilon}}$ \\
\hline$\varepsilon$ & $S S_{\varepsilon}=S S_{\text {Total }}-S S_{A}-S S_{B}-S S_{A B}-S S_{B l o c k}$ & $(a b-1)(n-1)$ & $M S_{\varepsilon}=\frac{S S_{\varepsilon}}{(a b-1)(n-1)}$ & \\
\hline Total & $S S_{\text {Total }}=\sum_{i} \sum_{j} \sum_{k} y_{i j k}^{2}-\frac{y_{\ldots}^{2}}{a b n}$ & $a b n-1$ & $M S_{\text {Total }}=\frac{S S_{\text {Total }}}{a b n-1}$ & \\
\hline
\end{tabular}

\section{Experimental Assessment}

The methodology was applied on a wind tunnel experiment of a ducted Boundary Layer Ingesting (BLI) propulsor. The experiment was conducted in the Low-Speed Tunnel (LST) operated by the German-Dutch Wind Tunnels (DNW). This atmospheric, closed circuit wind tunnel has a test section of $3.0 \mathrm{~m} \times 2.25 \mathrm{~m}$ cross section and length of $8.75 \mathrm{~m}$. The operating range of this wind tunnel is up to $80 \mathrm{~m} / \mathrm{s}$. The measurements were performed at a Mach number of 0.174 and a body length-based Reynolds number of $6 \times 10^{6}$ corresponding to a freestream velocity of $60 \mathrm{~m} / \mathrm{s}$. An axisymmetric body is placed upstream of the propulsor as shown in Fig. 1. The axisymmetric body has a length of $1.5 \mathrm{~m}$ and a maximum cross-sectional area of $0.0491 \mathrm{~m}^{2}$. The distance between the trailing edge of the body and the inlet of the propulsor is $0.15 \mathrm{~m}$. Stereoscopic PIV measurements were performed in a cross plane $0.08 \mathrm{~m}$ downstream from the outlet of the propulsor. The aim of this experimental assessment is to employ the statistical tools DOE and ANOVA to quantify the total uncertainty in time-averaged PIV measurements and the contribution of the design and nuisance factors to the total uncertainty. 


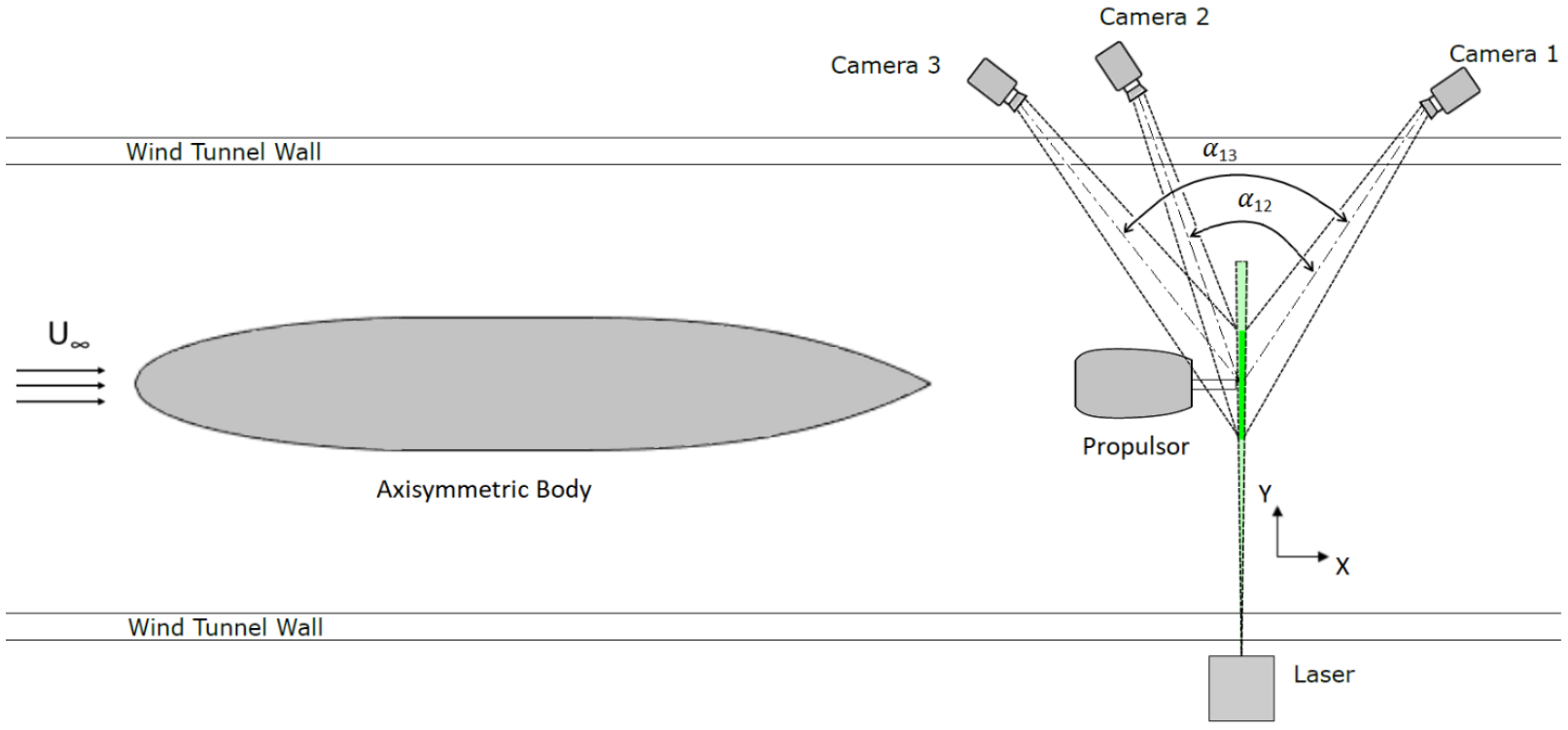

Figure 1: Schematic experimental setup of stereoscopic PIV measurements at the outlet of the ducted Boundary Layer Ingesting (BLI) propulsor

Various factors during the acquisition and processing stages contribute to the total uncertainty. However, only some of the most significant ones are considered for the analysis. Following Sciacchitano (2019) and Bhattacharya et al (2016), among others, four factors, namely camera aperture ( $f \#)$, inter-frame time separation $(\Delta t)$, interrogation window size $\left(D_{I}\right)$ and stereoscopic camera angle $(\alpha)$ were considered to be significant. Therefore, for the analysis three design factors- $f \#, \Delta t, D_{I}$ (assigned with $A, B, C$, respectively) and a blocking factor $(\alpha)$ with two levels of each were selected. The two levels of the factors were: 4 and 5.6 for $f \#, 16$ and $20 \mu$ s for $\Delta t, 16$ and 32 pixels for $D_{I}$, and 44 and 54 degrees for $\alpha$. Following the $2^{N}$ rule, $N$ being the number of design factors, a total of 16 measurements were performed $\left(2^{3}=8\right.$ in each block).

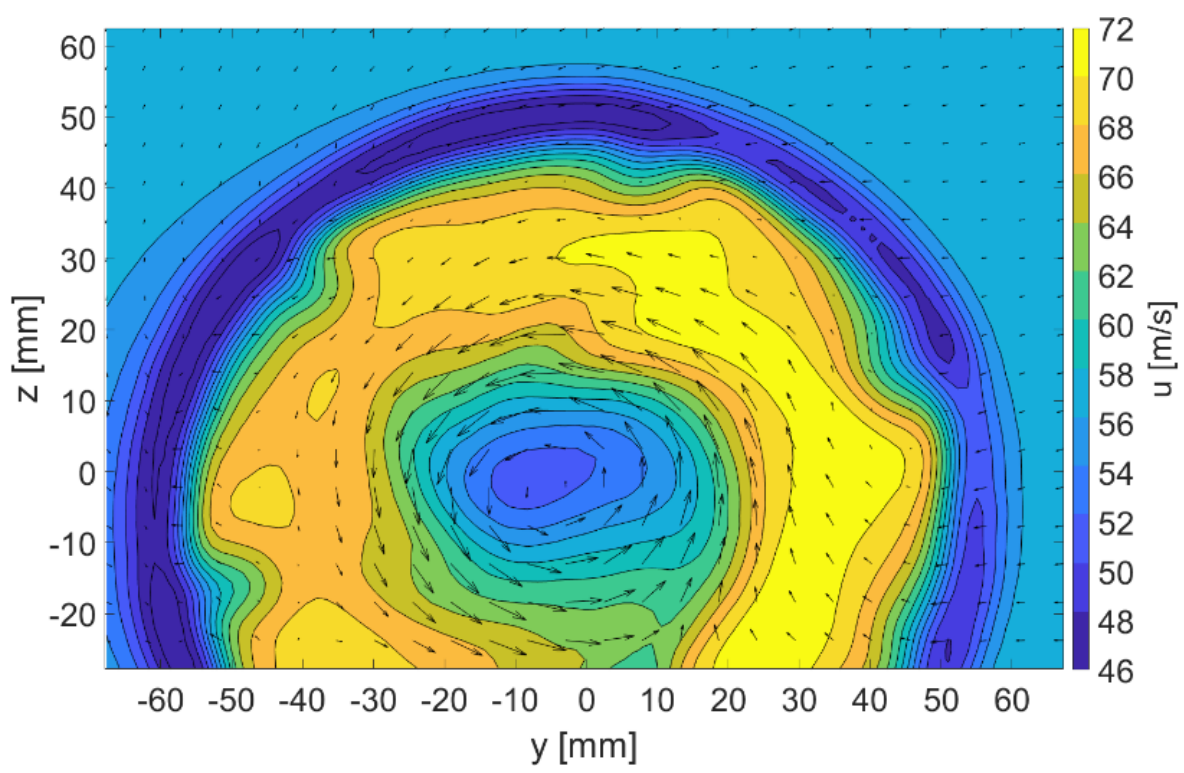

Figure 2: Time-averaged contour plots of the streamwise velocity $u$ and vector plots of the in-plane ( $Y$ - $Z$ plane) velocity of the stereoscopic PIV measurement 


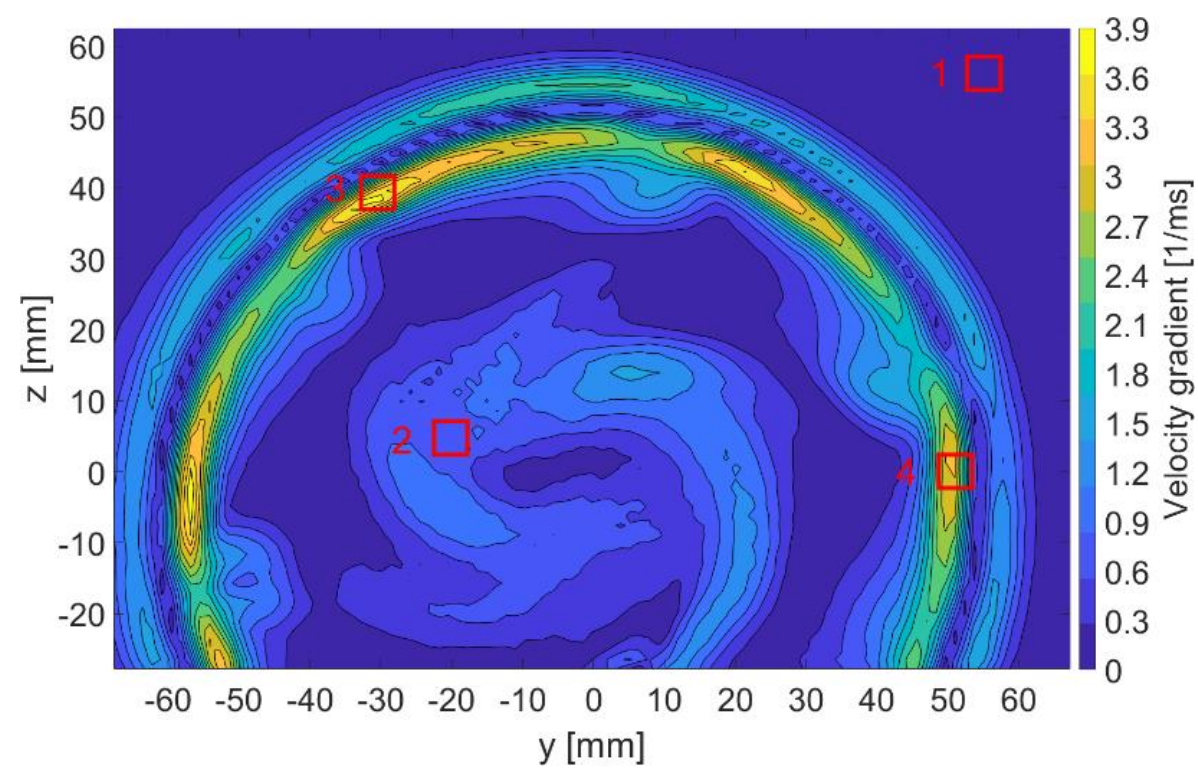

(a) Magnitude of local in-plane velocity gradient for streamwise velocity

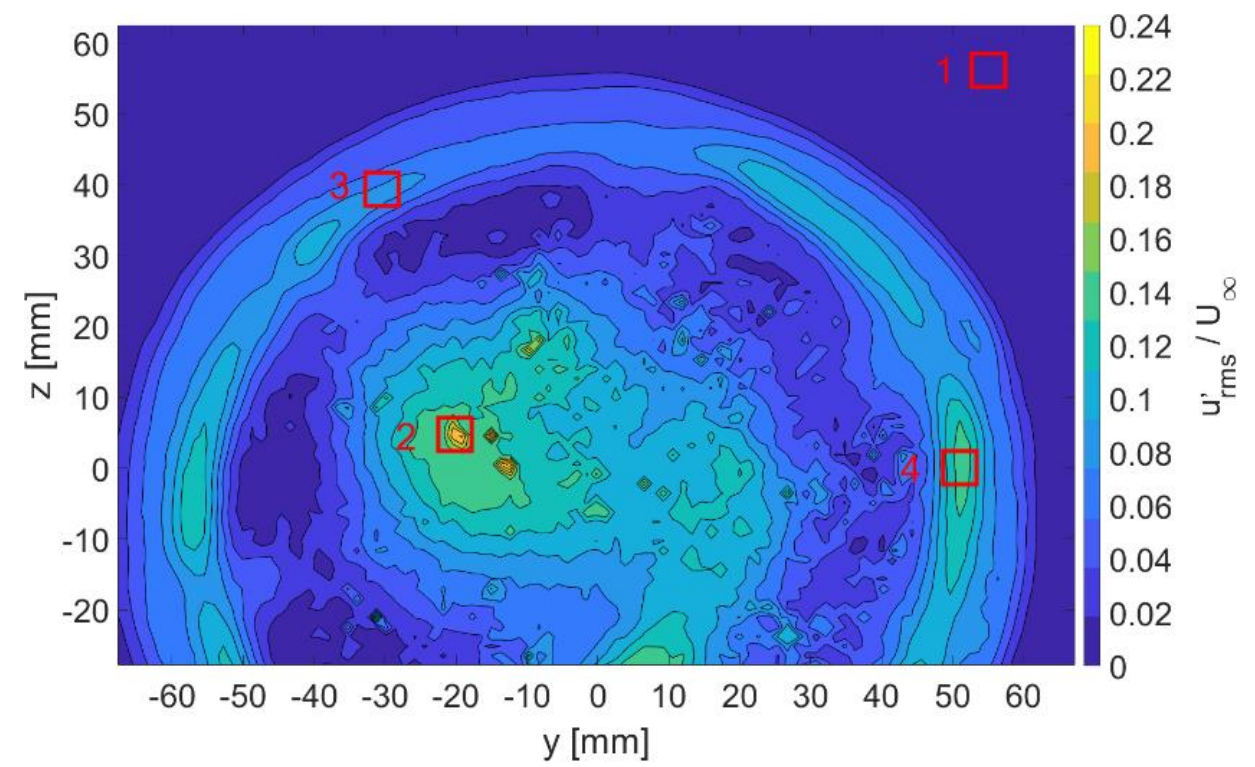

(b) RMS of velocity fluctuations in streamwise velocity normalized by freestream velocity

Figure 3: Contour plots of (a) magnitude of local in-plane velocity gradient for streamwise velocity and (b) RMS of velocity fluctuations in the streamwise velocity normalized by the freestream velocity (the detailed results of ANOVA are shown for the four regions which are selected based on the magnitude of velocity gradient and flow fluctuations: $1=$ low velocity gradient and low flow fluctuations, $2=$ low velocity gradient and high flow fluctuations, $3=$ high velocity gradient and low flow fluctuations, and $4=$ high velocity gradient and high flow fluctuations).

Three LaVision Imager sCMOS cameras were used to perform the measurements with two different stereoscopic angles (i.e. in two blocks). The cameras 1 and 2 formed the stereoscopic angle $\left(\alpha_{12}\right)$ of $44^{\circ}$ and were considered to be the block I, whereas the cameras 1 and 3 formed the stereoscopic angle $\left(\alpha_{13}\right)$ of $54^{\circ}$ and 
were considered to be the block II. It is to be noted that the stereoscopic angles were limited by the limited optical accessibility due to the wind tunnel wall and the position of the propulsor itself. The cameras have a sensor resolution of $2560 \times 2160$ pixels and a pixel size of $6.5 \mu \mathrm{m}$. The cameras were mounted with objective lenses of $135 \mathrm{~mm}$ focal length and Scheimpflug adapter. The field of view (FOV) obtained was $460 \mathrm{~mm} \times 220$ $\mathrm{mm}$ and the magnification factors for the cameras 1,2 and 3 were $0.067,0.073$ and 0.069 , respectively. The flow was seeded by an aerosol seeding generator, which produces DEHS droplets of $1 \mu \mathrm{m}$ mean diameter. The particles were illuminated by a Quantel Evergreen 200 laser (Nd:YAG, pulse energy of $200 \mathrm{~mJ}$ per pulse, wavelength of $532 \mathrm{~nm}$ ) forming a sheet of $4 \mathrm{~mm}$ thickness. The images were recorded and processed using the LaVision Davis 10.1.2 software. The data set at each run consisted of 2000 double-frame images and a total of 8 runs per block (stereoscopic camera angle) were performed in a random order. Each measurement run was unique corresponding to the combination of one of the two levels of the three design factors. The processing was done using Gaussian interrogation windows of $64 \times 64$ pixels with $50 \%$ overlap for the initial passes and $16 \times 16$ pixels or $32 \times 32$ pixels with $50 \%$ overlap for the final passes.

The estimated time-averaged streamwise velocity component $u$ and in-plane ( $Y$ - $Z$ plane) velocity vectors are shown in Fig. 2. The wake region can be seen in the centre of the measurement domain, whereas the outer region represents potential flow with streamwise velocity of $60 \mathrm{~m} / \mathrm{s}$. The flow is retarded at the periphery of the propulsor and the discontinuities in the mean streamwise velocity field due to the stator ring can be seen at the periphery. Moreover, the in-plane velocity vectors are shown in Fig. 2 which represent the magnitude and direction of $Y$ and $Z$-velocity components $v$ and $w$, respectively. The counter-clockwise rotation of the flow in the wake of the propulsor can be easily seen due to the direction of the vectors, where the magnitudes of $v$ and $w$ velocity components are larger than those in the outer potential flow region. Moreover, contour plots of magnitude of local in-plane velocity gradient for streamwise velocity and RMS of fluctuations in the streamwise velocity normalized by the freestream velocity $\left(U_{\infty}\right)$ are shown in Fig. 3(a) and (b), respectively. It is clear that the flow has varying degrees of velocity gradients and velocity fluctuations. Therefore, the measured flow field is a suitable case to implement and assess the feasibility of the proposed approach in a range of flow conditions encountered in typical PIV measurements. The analysis was performed for the whole FOV to quantify the total uncertainty in the time-averaged velocity and the contribution of the individual factors to the total uncertainty. However, for simplicity, four regions were chosen based on the amount of velocity gradient and flow fluctuations, as shown in Fig. 3(a) and (b), to explain the contribution of the factors to the total uncertainty. The results at these selected regions are explained in detail in Section 4.

\section{Results}

The application of the proposed methodology to the flow at the outlet of the BLI propulsor resulted into the quantification of the total uncertainty in the time-averaged velocity components. The contour plot of the total uncertainty $\left(U_{u}\right)$ of the mean streamwise velocity component $u$ is shown in Fig. 4. As expected, the total uncertainty of the mean velocity closely resembles the fluctuations root-mean-square, because it is equal to $\sigma / \sqrt{N_{s}}$ ( $\sigma$ being the standard deviation and $N_{s}$ the number of samples), as discussed by Sciacchitano and Wieneke (2016). Moreover, larger total uncertainty is retrieved in the regions of high velocity gradients, as reported by Scarano (2002), which are mainly encountered at the outer edge of the propulsor slipstream. Following these observations, four small regions in the flow field were selected to evaluate the results for the constituent systematic uncertainties based on the amount of velocity gradient and flow fluctuations as shown in Fig. 3(a) and (b). The regions 1, 2, 3 and 4 correspond to the regions of low velocity gradient and low flow fluctuations, low velocity gradient and high flow fluctuations, high velocity gradient and low flow fluctuations, and high velocity gradient and high flow fluctuations, respectively, as shown in the table 2.

The ANOVA results in the four regions are obtained in the form of tables like the table 1 , where the $F_{0}$ values corresponding to the main and interaction effects of the design and blocking factors are calculated as shown in 
the last column in the table 1 . The F-test is then performed to estimate whether the effects are statistically significant or not, which is done by comparing the $F_{0}$ values with the critical value $F_{c}$ which is 5.3 for $95 \%$ confidence level (for 1 degree of freedom of numerator and 8 degrees of freedom of denominator) in the present experimental case. If the $F_{0}$ value is greater than $F_{c}$, then the corresponding effect is statistically significant with the desired level of confidence. For example, in the region 3 in the measurement domain, the main effect of factor B (i.e. $\Delta t$ ) is statistically significant as it shows $F_{0}$ value of 11.5. The reader is advised to refer any standard book of statistics for a detailed explanation of the F-test (for example, Montgomery 2013). The constituent uncertainties due to the main and interaction effects of the factors are calculated by equation (3) and their contributions to the total uncertainty in the streamwise velocity $u$ are shown in the form of pie charts in Fig. 5. The pie charts represent the percentage contribution of the systematic uncertainties to the total uncertainty. The sub-figures (a), (b), (c) and (d) are for the regions 1, 2, 3 and 4, respectively, which are marked in Fig. 3(a) and (b). The mean streamwise velocity $(u)$ components in these regions are $59.20 \mathrm{~m} / \mathrm{s}$, $59.75 \mathrm{~m} / \mathrm{s}, 52.28 \mathrm{~m} / \mathrm{s}$ and $64.17 \mathrm{~m} / \mathrm{s}$, respectively. The corresponding total uncertainties are $0.37 \mathrm{~m} / \mathrm{s}, 0.51$ $\mathrm{m} / \mathrm{s}, 1.56 \mathrm{~m} / \mathrm{s}$ and $1.60 \mathrm{~m} / \mathrm{s}$, which are shown in the centre of the pie charts in Fig. 5 .

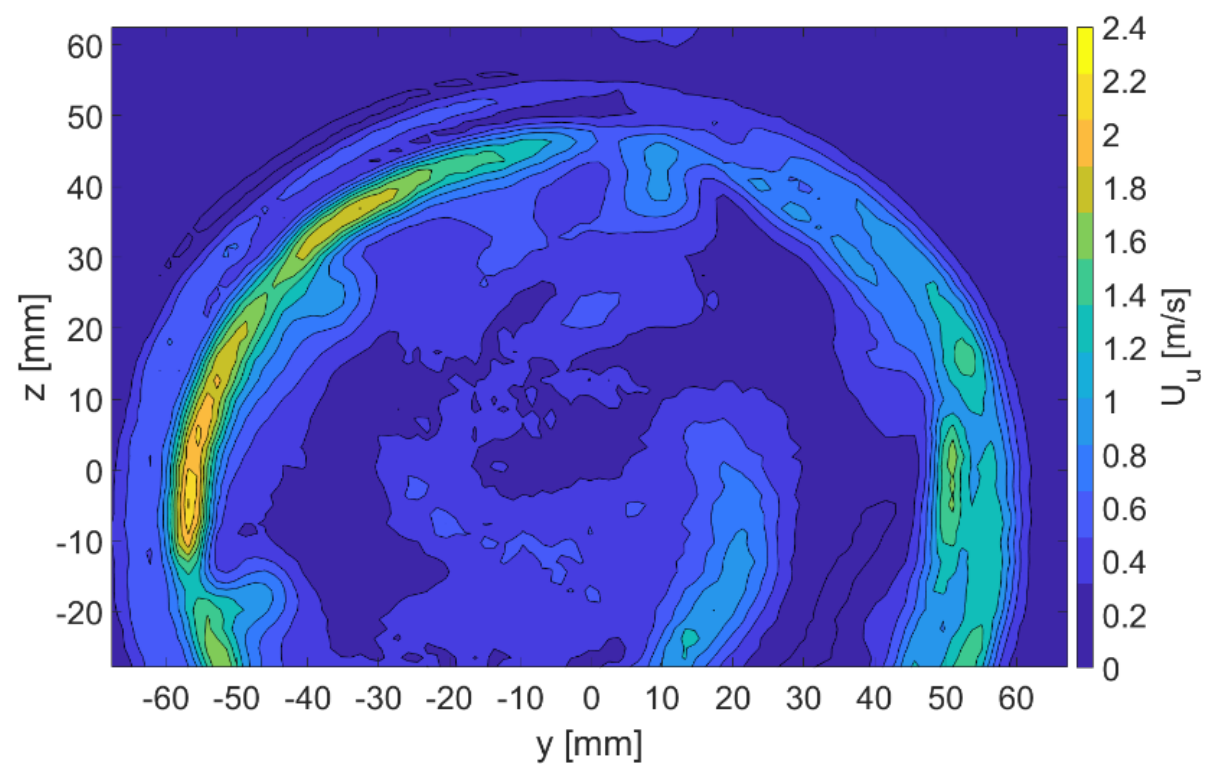

Figure 4: Total uncertainty in time-averaged streamwise velocity $u$ calculated by the proposed methodology

Table 2: Magnitude of local in-plane velocity gradient for streamwise velocity and RMS of velocity fluctuations in the streamwise velocity $\left(u^{\prime}{ }_{r m s}\right)$ normalized by the freestream velocity $\left(U_{\infty}\right)$ at the four regions of analysis marked in Fig. 3(a) and (b)

\begin{tabular}{ccc}
\hline Regions & Velocity gradient $(\mathbf{1} / \mathbf{m s})$ & $\boldsymbol{u}_{r m s}^{\prime} / \boldsymbol{U}_{\infty}$ \\
\hline 1 & $0.00-0.02$ & $0.00-0.01$ \\
2 & $0.70-0.80$ & $0.18-0.22$ \\
3 & $2.70-3.90$ & $0.06-0.08$ \\
4 & $2.70-3.30$ & $0.14-0.16$ \\
\hline
\end{tabular}




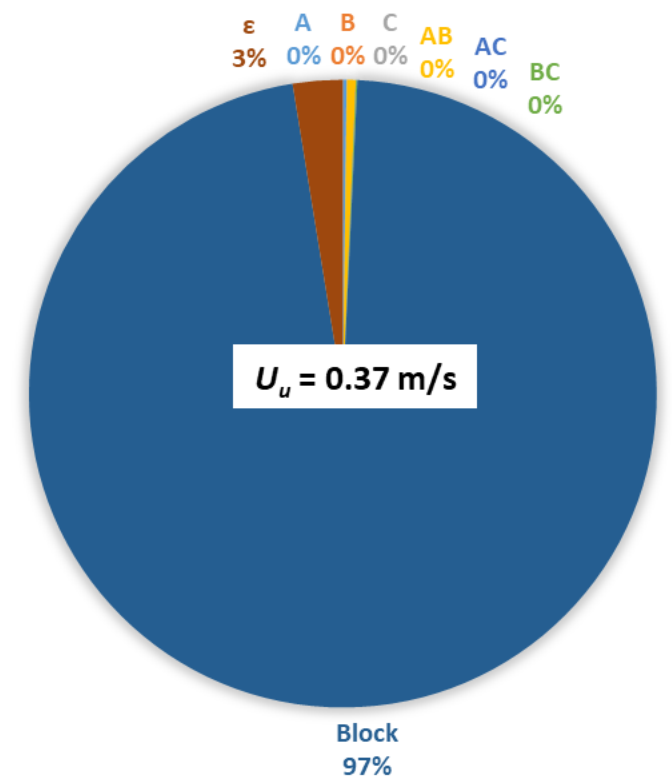

(a) Region 1

(low velocity gradient and low flow fluctuations)

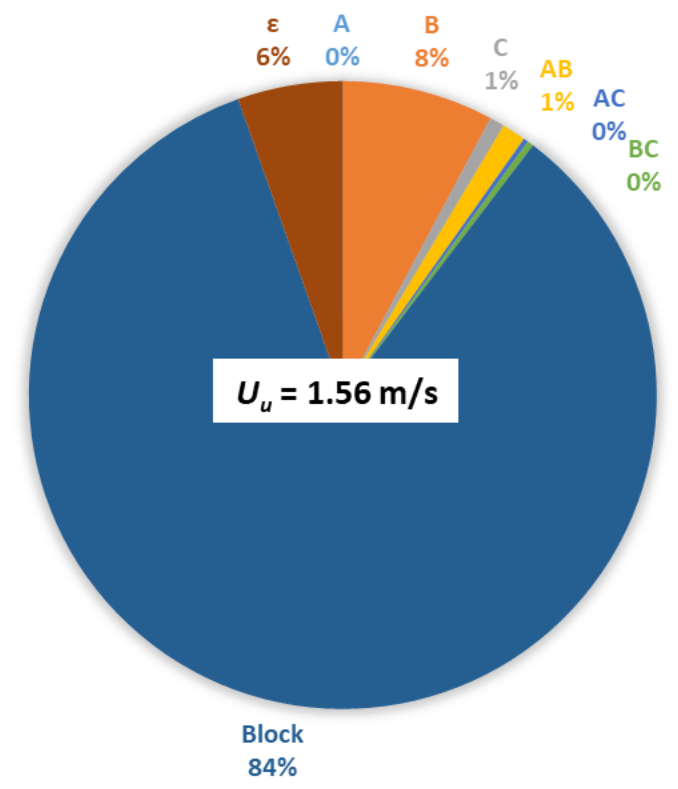

(c) Region 3

(high velocity gradient and low flow fluctuations)

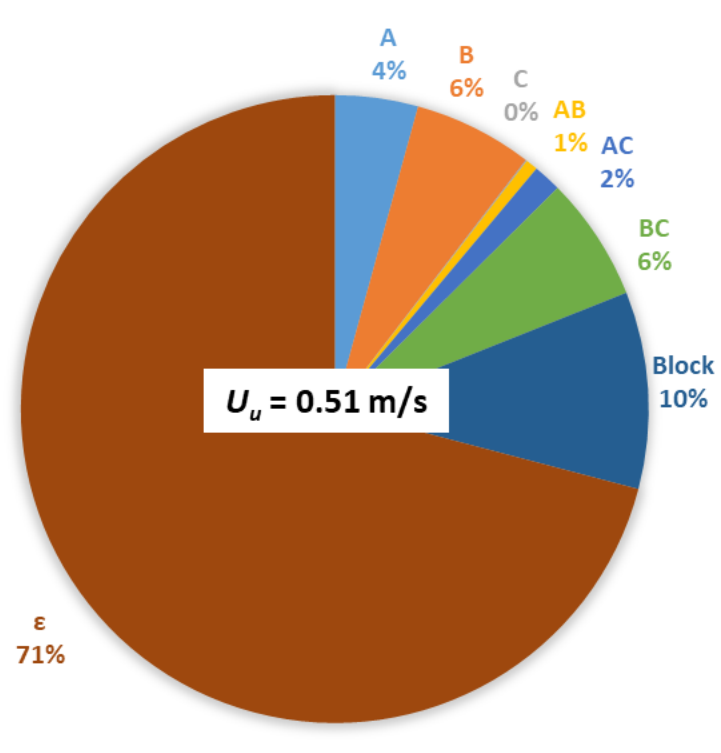

(b) Region 2

(low velocity gradient and high flow fluctuations)

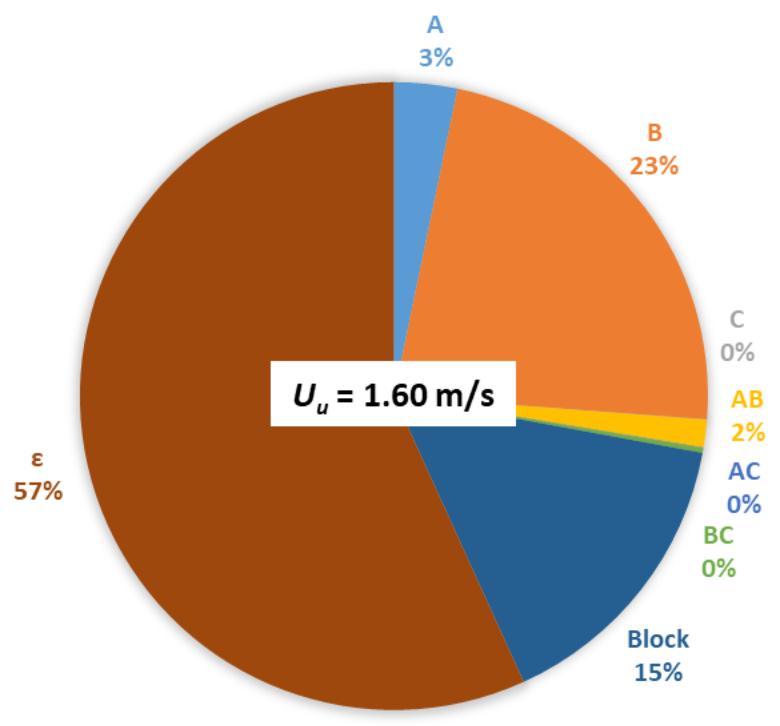

(d) Region 4

(high velocity gradient and high flow fluctuations)

Figure 5: Contribution of systematic uncertainties to the total uncertainty in time-averaged streamwise velocity for the regions marked in Fig. 3(a) and (b), due to main and interaction effects of the factors- $A$ (camera aperture $f \#$ ), $B$ (inter-frame time separation $\Delta t$ ), $C$ (interrogation window size $D_{I}$ ) and block of stereoscopic camera angle

It is clear that the total uncertainty increases with increase in the velocity gradient and the flow fluctuations, which agrees with the observation in the contour plot of total uncertainty in Fig 4. The analysis shows that, in 
most of the measurement domain, the main effects of the factors $f \#$ (factor $A$ ), $\Delta t$ (factor $B$ ) and stereoscopic camera angle (block) are statistically significant, whereas the main effect of the factor $D_{I}$ (factor $C$ ) and all the two-way interaction effects are found to be insignificant. The blocking factor- stereoscopic camera angle is the most significant factor in all of the four regions. It is due to the slight misalignment of the two measurement planes of the two blocks of the measurements (viz. the two stereoscopic systems). However, this uncertainty corresponding to the stereoscopic camera angles can be easily reduced by implementing a proper self-calibration (Wieneke 2005), which has not been applied in this study. Nevertheless, the stereoscopic camera angle is highly influential in stereoscopic PIV measurements (Prasad 2000) and should be selected optimally to minimize the related errors. The factor $\Delta t$ corresponds to the out-of-plane motion of the particles and it has significant contribution to the total uncertainty of the time-averaged streamwise velocity component in the regions of high velocity gradients and high fluctuations (regions 2, 3 and 4). However, the analysis for $Y$ and $Z$ velocity components show that the factor $\Delta t$ is significant also in the regions of low flow fluctuations (the results are not shown for conciseness). In that case, the factor $\Delta t$ corresponds to peak-locking error and, as observed by Adatrao et al. (2021), the regions of low flow fluctuations are those where the mean velocity is affected by peak-locking errors the most. Additionally, the factor $f \#$ is associated with errors due to out-offocus particles caused by imperfect camera focussing. Analysing the image recordings, it was realized that, at the low level of the $f \#$, the particle images in the potential flow in the top-left region of measurement domain for the camera 2 were out of focus, thus yielding larger uncertainty. For the flow regions of low flow fluctuations, i.e. regions 1 and 3, the blocking factor (i.e. the stereoscopic camera angle) contributes the most ( $97 \%$ and $84 \%$, respectively) to the total uncertainty in the time-averaged velocity. However, for the flow regions of high flow fluctuations, i.e. the regions 2 and 4, the random error (factors not directly considered in the analysis, e.g. limited statistical convergence, image noise, variations of seeding concentration, etc.) has the biggest contribution of $71 \%$ and $57 \%$ to the total uncertainty in the mean streamwise velocity, as shown in Fig. 5(b) and (d), respectively. This is due to the flow fluctuations in these regions being high which, owed to the limited statistical convergence of the measurements, makes it difficult to segregate the contribution of individual systematic uncertainties. It is to be noted that the error uncertainty from the ANOVA represents the random uncertainty in the measurements plus the uncertainty due to the unknown experimental factors (i.e. the factors not considered as design or blocking factors).

\section{Conclusions}

A PIV uncertainty quantification (UQ) approach is proposed based on a statistical tool called Design of Experiments (DOE). The basic principle of the approach is to measure a constant (ideally) quantity for the different levels of experimental factors and to compute total variance and individual variances arising from the different levels of each of the factors. The statistical analysis is performed using Analysis of Variances (ANOVA). The proposed methodology is assessed considering the stereoscopic PIV measurements of a flow at the outlet of a Boundary Layer Ingesting (BLI) propulsor to quantify the uncertainty of the time-averaged velocity. Three design factors, namely camera aperture $(f \#)$, inter-frame time separation $(\Delta t)$ and interrogation window size $\left(D_{I}\right)$, and a blocking factor (the stereoscopic camera angle $\alpha$ ) are considered for the analysis. The three design factors and the blocking factor are set at two different levels (low and high) each, resulting in a total of 16 measurements $\left(2^{3}=8\right.$ in each block, following the $2^{N}$ rule, $N$ being the number of design factors) which are performed in a random order. The analysis results in the estimation of total uncertainty in the timeaveraged velocity as well as constituent systematic uncertainties due to the design and blocking factors. Overall, it can be concluded that the stereoscopic camera angle has the largest contribution to the uncertainty of the measured time-averaged velocity (without self-calibration). The random errors due to the unknown experimental factors and limited statistical convergence are significant especially in regions of high fluctuations. Of the design factors considered in this study, the inter-frame time separation $\Delta t$ is highly significant especially in the regions of high flow fluctuations for the streamwise velocity component, whereas 
it is significant for the velocity components $v$ and $w$ in the regions of high velocity gradients. A marginal role is played by the $f \#$, due to the presence of out-of-plane particles in a region of camera 2 . The contribution of the window size and of the interactions among factors to the uncertainty of the time-averaged velocity is found not to be statistically significant. The present work is thus able to segregate the systematic uncertainties due to the experimental factors considered for the analysis. Moreover, knowing these constituent uncertainties, it will be possible to optimize the experiment in order to reduce the total uncertainty. The proposed methodology is successfully used for the time-averaged velocity. However, it can also be applied to the higher order statistics, e.g. Reynolds stresses, to quantify the total and constituent systematic uncertainties in those quantities. Moreover, the approach can easily be implemented for 3D measurements for uncertainty quantification. The future work will focus on validating and comparing the proposed approach with the conventional PIV-UQ approaches. Additionally, a comparison between the $2^{N}$ and $3^{N}$ models (two and three levels of the design factors, respectively) will be performed. It is also planned to perform the self-calibration before applying the proposed methodology for the stereoscopic PIV measurements.

\section{Acknowledgements}

The research is partly funded by the Dutch Research Organization NWO domain Applied and Engineering Sciences, Veni grant 15854 Deploying Uncertainty Quantification in Particle Image Velocimetry.

\section{References}

Adatrao S, Bertone M and Sciacchitano A (2021) Multi- $\Delta t$ approach for peak-locking error correction and uncertainty quantification in PIV. Meas. Sci. Technol. 32:054003

Beresh SJ (2009) Comparison of PIV data using multiple configurations and processing techniques. Experiments in Fluids 47:883-896

Bhattacharya S, Charonko JJ and Vlachos PP (2016) Stereoscopic-particle image velocimetry uncertainty quantification. Meas. Sci. Technol. 28:015301

DeBonis JR, Oberkampf WL, Wolf RT, Orkwis PD, Turner MGBH and Benek JA (2012) Assessment of computational fluid dynamics and experimental data for shock boundary-layer interactions. AIAA Journal 50:891-903

DeLoach R (2000) The modern design of experiments: a technical and marketing framework. in 21st AIAA Advanced Measurement Technology and Ground Testing Conference, Denver, CO, USA, June 19-22

DeLoach R, Obara CJ and Goodman W (2012) A practical methodology for quantifying the random and systematic components of unexplained variance in a wind tunnel. in 50th AIAA Aerospace Sciences Meeting and Exhibit, Nashville, Tennessee, USA, January 9-12

Kähler CJ, Astarita T, Vlachos PP, Sakakibara J, Hain R, Discetti S, Foy R and Cierpka C (2016) Main results of the 4th international PIV challenge. Experiments in Fluids 57:1-71

Montgomery DC (2013) Design and analysis of experiments. 8th Edition, John Wiley \& Sons, New York

Prasad AK (2000) Stereoscopic particle image velocimetry. Experiments in Fluids 29:103-116

Scarano F (2002) Iterative image deformation methods in PIV. Meas. Sci. Technol. 13:R1-R19

Sciacchitano A (2019) Uncertainty quantification in particle image velocimetry. Meas. Sci. Technol. 30:092001

Sciacchitano A and Wieneke B (2016) PIV uncertainty propagation. Meas. Sci. Technol. 27:084006

Smith BL and Oberkampf WL (2014) Limitations of and alternatives to traditional uncertainty quantification for measurements. in the ASME 2014 Fluids Engineering Summer Meeting, Chicago, IL, USA, August 3-7

Wieneke B (2005) Stereoscopic-PIV using self-calibration on particle images. Experiments in Fluids 39:267-280 